\title{
Uniqueness of Embedding into a Gaussian Semigroup and a Poisson Semigroup with Determinate Jump Law on a Simply Connected Nilpotent Lie Group
}

\section{Daniel Neuenschwander}

Received: 16 March 2007 / Revised: 19 May 2008 / Published online: 13 September 2008

(C) Springer Science+Business Media, LLC 2008

Abstract Let $\left\{\mu_{t}^{(i)}\right\}_{t \geq 0}(i=1,2)$ be continuous convolution semigroups (c.c.s.) on a simply connected nilpotent Lie group $G$. Suppose that $\mu_{1}^{(1)}=\mu_{1}^{(2)}$. Assume furthermore that one of the following two conditions holds:

(i) The c.c.s. $\left\{\mu_{t}^{(1)}\right\}_{t \geq 0}$ is a Gaussian semigroup (in the sense that its generating distribution just consists of a primitive distribution and a second-order differential operator)

(ii) The c.c.s. $\left\{\mu_{t}^{(i)}\right\}_{t \geq 0}(i=1,2)$ are both Poisson semigroups, and the jump measure of $\left\{\mu_{t}^{(1)}\right\}_{t \geq 0}$ is determinate (i.e., it possesses all absolute moments, and there is no other nonnegative bounded measure with the same moments).

Then $\mu_{t}^{(1)}=\mu_{t}^{(2)}$ for all $t \geq 0$. As a complement, we show how our approach can be directly used to give an independent proof of Pap's result on the uniqueness of the embedding Gaussian semigroup on simply connected nilpotent Lie groups. In this sense, our proof for the uniqueness of the embedding semigroup among all c.c.s. of a Gaussian measure can be formulated self-contained.

Dedicated to Professor Wilfried Hazod on the occasion of his 65th birthday.

D. Neuenschwander $(\bowtie)$

École des Hautes Etudes Commerciales, Institut de Sciences Actuarielles, Université de Lausanne, 1015 Lausanne, Switzerland

e-mail: daniel.neuenschwander@bluewin.ch

D. Neuenschwander

Institut für mathematische Statistik und Versicherungslehre, Universität Bern, 3012 Bern,

Switzerland

D. Neuenschwander

Institut de Science Financière et d'Assurances, Université de Lyon, Université Claude Bernard Lyon 1, 50 avenue Tony Garnier, 69007 Lyon, France 
Keywords Continuous convolution semigroups · Simply connected nilpotent Lie groups $\cdot$ Gaussian semigroups $\cdot$ Poisson semigroups

Mathematics Subject Classification (2000) Primary 60B15 · Secondary 60G99 . $22 \mathrm{E} 25$

\section{Introduction}

Let $G$ be a locally compact group, $e$ the neutral element, and $G^{*}:=G \backslash\{e\}$. The structure $\left(M^{1}(G), *, \stackrel{w}{\rightarrow}\right)$ is the topological semigroup of (regular) probability measures on $G$ equipped with operation of convolution and weak topology (see [10], Theorem 1.2.2). A continuous convolution semigroup $\left\{\mu_{t}\right\}_{t \geq 0}$ of probability measures on $G$ (c.c.s. for short) is a continuous semigroup homomorphism

$$
\begin{aligned}
& \left(\left[0, \infty[,+) \ni t \mapsto \mu_{t} \in\left(M^{1}(G), *, \stackrel{w}{\rightarrow}\right),\right.\right. \\
& \mu_{0}=\varepsilon_{e}
\end{aligned}
$$

( $\varepsilon_{x}$ denoting the Dirac probability measure at $x \in G$ ). For simply connected nilpotent Lie groups, the requirement $\mu_{0}=\varepsilon_{e}$ is no restriction, since in any case $\mu_{0}$ must be an idempotent element of $M^{1}(G)$ and thus is the Haar measure $\omega_{K}$ on some compact subgroup $K \subset G$ (see [10], 1.5.6); however, simply connected nilpotent Lie groups have no nontrivial compact subgroups (see [16], 2.2). Let $G$ be a Lie group, $C_{b}^{\infty}(G)$ the space of bounded complex-valued $C^{\infty}$-functions on $G$, and $\mathcal{D}(G)$ the subspace of complex-valued $C^{\infty}$-functions with compact support.

The generating distribution $\mathcal{A}$ of a c.c.s. $\left\{\mu_{t}\right\}_{t \geq 0}$ is defined (for $f \in \mathcal{D}(G)$ ) as

$$
\begin{aligned}
\mathcal{A} f & :=\lim _{t \rightarrow 0+} \frac{1}{t} \int_{G}[f(x)-f(e)] \mu_{t}(d x) \\
& =\left.\frac{d}{d t}\right|_{t=0+} \int_{G} f(x) \mu_{t}(d x) \quad(f \in \mathcal{D}(G)) .
\end{aligned}
$$

It exists on the whole of $C_{b}^{\infty}(G)$ (see [21], p. 119). Now let $G$ be a simply connected nilpotent Lie group. This means that $G$ is a Lie group with Lie algebra $\mathcal{G}$ such that $\exp : \mathcal{G} \rightarrow G$ is a diffeomorphism and that the descending central series is finite, i.e., there is $r \in \mathbb{N}_{0}$ such that

$$
\mathcal{G}_{0} \supsetneqq \mathcal{G}_{1} \supsetneqq \cdots \supsetneqq \mathcal{G}_{r}=\{0\},
$$

where

$$
\mathcal{G}_{0}:=\mathcal{G}, \quad \mathcal{G}_{k+1}:=\left[\mathcal{G}, \mathcal{G}_{k}\right] \quad(0 \leq k \leq r-1) .
$$

The group $G$ is then called step $r$-nilpotent. We further identify $G$ with $\mathcal{G}=\mathbb{R}^{d}$ via $\log$ (the inverse map of exp). So $G$ may be interpreted as $\mathbb{R}^{d}$ equipped with a Lie bracket [., .] : $\mathbb{R}^{d} \times \mathbb{R}^{d} \rightarrow \mathbb{R}^{d}$, which is bilinear, skew-symmetric, and satisfies the Jacobi identity

$$
[[x, y], z]+[[y, z], x]+[[z, x], y]=0 .
$$


Write $\operatorname{ad}(x)():.=[x,$.$] for the adjoint map on \mathcal{G}$. The group product is then given by the Campbell-Hausdorff formula (see [19] and [11], p. 9), where due to the nilpotency only the terms up to order $r$ arise:

$$
\begin{aligned}
& x \cdot y=\sum_{n=1}^{r} z_{n}, \\
& z_{n}=\frac{1}{n} \sum_{p+q=n}\left(z_{p, q}^{\prime}+z_{p, q}^{\prime \prime}\right) \text {, } \\
& z_{p, q}^{\prime}=\sum_{\substack{p_{1}+p_{2}+\cdots+p_{m}=p \\
q_{1}+q_{2}+\cdots+q_{m-1}=q-1 \\
p_{i}+q_{i} \geq 1 \\
p_{m} \geq 1}} \frac{(-1)^{m+1}}{m} \frac{\operatorname{ad}(x)^{p_{1}}\left(\operatorname{ad}(y)^{q_{1}}\left(\cdots \operatorname{ad}(x)^{p_{m}}(y)\right) \cdots\right)}{p_{1} ! q_{1} ! \cdots p_{m} !}, \\
& z_{p, q}^{\prime \prime}=\sum_{\substack{p_{1}+p_{2}+\cdots+p_{m-1}=p-1 \\
q_{1}+q_{2}+\cdots+q_{m-1}=q \\
p_{i}+q_{i} \geq 1}} \frac{(-1)^{m+1}}{m} \frac{\operatorname{ad}(x)^{p_{1}}\left(\operatorname{ad}(y)^{q_{1}}\left(\cdots \operatorname{ad}(y)^{q_{m-1}}(x)\right) \cdots\right)}{p_{1} ! q_{1} ! \cdots q_{m-1} !} .
\end{aligned}
$$

The first few terms are

$$
x \cdot y=x+y+\frac{1}{2}[x, y]+\frac{1}{12}([[x, y], y]+[[y, x], x])+\cdots .
$$

It is clear that the neutral element $e$ is 0 and that the inverse element of $x \in G$ is $-x$. The generating distribution of a c.c.s. on $G$ assumes a very explicit form: The functional $\mathcal{A}$ on $C_{b}^{\infty}(G)$ is a generating distribution of a c.c.s. $\left\{\mu_{t}\right\}_{t \geq 0}$ iff it has the form (Lévy-Hinčin formula)

$$
\mathcal{A} f=\langle\xi, \nabla\rangle f(0)+\frac{1}{2}\langle\nabla, M \cdot \nabla\rangle f(0)+\int_{G^{*}}[f(x)-f(0)-\Psi(f, x)] \eta(d x),
$$

where

$$
\Psi(f, x):= \begin{cases}\langle x, \nabla\rangle f(0), & \|x\| \leq 1, \\ \left\langle\frac{x}{\|x\|}, \nabla\right\rangle f(0), & \|x\|>1\end{cases}
$$

$\left(f \in C_{b}^{\infty}(G)\right), \xi \in G \cong \mathcal{G} \cong \mathbb{R}^{d}, M$ is a positive semidefinite $d \times d$-matrix, and $\eta$ is a Lévy measure on $G^{*}$, i.e., a nonnegative measure on $G^{*}$ satisfying

$$
\int_{0<\|x\| \leq 1}\|x\|^{2} \eta(d x)+\eta(\{x \in G:\|x\|>1\})<\infty .
$$

The first summand in the Lévy-Hinčin formula is called the primitive term, the second one the centered Gaussian term, and the third one (the integral expression) the generalized Poisson distribution. The data $\xi, M, \eta$ are uniquely determined by $\left\{\mu_{t}\right\}_{t \geq 0}$ (see [20], Satz 1). As a shorthand, we will write $\mathcal{A}=[\xi, M, \eta]$. In particular, we have that $\left.\left.(1 / t) \mu_{t}\right|_{B} \stackrel{w}{\rightarrow} \eta\right|_{B}(t \rightarrow 0)$ for every open subset $B$ of $G$ bounded away 
from 0 (this immediately follows from the definition of the generating distribution). The distribution $\mathcal{A}$ on $C_{b}^{\infty}(G)$ uniquely determines the c.c.s. $\left\{\mu_{t}\right\}_{t \geq 0}$; for this reason, we may write $\mu_{t}=: \operatorname{Exp} t \mathcal{A}(t \geq 0)$. Furthermore, every triple $[\xi, M, \eta]$ of the abovementioned type generates a c.c.s. (see [20], Satz 1). The generating distribution $\mathcal{A}$ is called Gaussian if $\eta=0$. A c.c.s. $\{\operatorname{Exp} t \mathcal{A}\}_{t \geq 0}$ is called Gaussian if $\mathcal{A}$ is Gaussian. A c.c.s. is called non-Gaussian if its generating distribution is not a Gaussian one, i.e., iff its Lévy measure is nonzero. A probability measure $\mu \in M^{1}(G)$ is called Gaussian if it is embeddable into a Gaussian c.c.s., i.e., if $\mu=\mu_{1}$ for some Gaussian c.c.s. $\left\{\mu_{t}\right\}_{t \geq 0}$. The objects $\{\operatorname{Exp} t \mathcal{A}\}_{t \geq 0}$ and $\operatorname{Exp} t \mathcal{A}$ are called Poissonian if

$$
\{\operatorname{Exp} t \mathcal{A}\}_{t \geq 0}=\left\{\exp \left(t\left(\eta-\|\eta\| \varepsilon_{0}\right)\right)\right\}_{t \geq 0}=\left\{e^{-t\|\eta\|} \exp (t \eta)\right\}_{t \geq 0}
$$

for some nonnegative bounded measure $\eta$ on $G$. Here exp denotes the usual exponential power series on the Banach algebra of bounded signed measures $\theta$ on $G$ (which is convergent in the topology induced by the norm of total variation):

$$
\exp \theta:=\varepsilon_{0}+\sum_{k=1}^{\infty}(1 / k !) \theta^{* k} .
$$

The measure $\eta$ will be called the exponent measure of the Poissonian semigroup. It is the law of jumps (w.l.o.g. assumed to be nonzero with the exception of the trivial case $\eta=0$ ) multiplied by the intensity parameter of the underlying Poisson counting process. The Lévy measure is thus just the restriction of the exponent measure to $G^{*}$. A $G$-valued random variable $X$ is called Gaussian resp. Poissonian if its law $\mathcal{L}(X) \in M^{1}(G)$ is Gaussian resp. Poissonian.

It has been shown by Burrell and McCrudden [3] that every infinitely-divisible probability measure on a simply connected nilpotent Lie group $G$ is embeddable into a c.c.s. on $G$ (like on finite-dimensional vector spaces). But now the question of uniqueness of the embedding c.c.s. (which is well known to hold on finitedimensional vector spaces) also is of great importance: If a sequence of ("approximating") c.c.s. converges for time $t=1$ to a limit measure which is embeddable into a unique ("limit") c.c.s., then the sequence of approximating c.c.s. converges to the limit c.c.s. as a whole (i.e., for all $t \geq 0$, even uniformly on compact subsets of the time axis). This is equivalent to the convergence of the corresponding generating distributions (for all $f \in C_{b}^{\infty}(G)$ ). By the "Poisson Approximation Theorem" (also called "Accompanying Laws Theorem") on $G$, it follows that if $\mu_{1}$ is embeddable into a unique c.c.s. $\left\{\mu_{t}\right\}_{t \geq 0}$ with generating distribution $\mathcal{A}$, then for a strictly increasing sequence $\{k(n)\}_{n \geq 1}$ of natural numbers and some sequence $\left\{v_{n}\right\} \subset M^{1}(G)$, the relation $v_{n}^{* k(n)} \stackrel{w}{\rightarrow} \mu_{1}(n \rightarrow \infty)$ implies $v_{n}^{*\lfloor k(n) t\rfloor} \stackrel{w}{\rightarrow} \mu_{t}(n \rightarrow \infty)$ $(t \geq 0)$; again, the latter is equivalent to the convergence of the Poisson generators $k(n) \int_{G}(f(x)-f(0)) v_{n}(d x) \rightarrow \mathcal{A}(f)$ as $n \rightarrow \infty\left(f \in C_{b}^{\infty}(G)\right)$ (see [8, 9, 11], [16, Remark 2.(a)] and the literature cited there). Finite groups satisfy the uniqueness property iff every nonneutral element has order 2 (then the group is of course Abelian) (see [2]). For locally compact Abelian groups, a sufficient condition for the uniqueness property is the requirement that the group has no nontrivial compact subgroup (see [10], Theorem 3.5.15). For irreducible symmetric spaces $G / K$ of noncompact 
type (i.e., $G$ a semisimple noncompact Lie group with finite center and $K$ a maximal compact subgroup) and $K$-biinvariant probability measures $\mu$ on $G$, Graczyk [6] used a method to associate to $\mu$ a bounded nonnegative measure $\check{\mu}$ on a Cartan subalgebra $(\mathbf{a},+)$ such that $\mu_{1} * \mu_{2}=\mu_{3}$ iff $\check{\mu}_{1} * \breve{\mu}_{2}=\breve{\mu}_{3}$ and such that $\check{\mu}$ determines $\mu$ uniquely. This readily yields the uniqueness property for all c.c.s. of $K$-biinvariant probability measures on $G$ by the uniqueness property on $(\mathbf{a},+)$. In a more general framework, some partial results have been obtained by Hazod [7]. For stable and semistable semigroups on simply connected nilpotent Lie groups, see $[8,9,16]$. The case of Poisson semigroups with boundedly supported Lévy measures on simply connected nilpotent Lie groups was addressed in [12]. Pap [17] proved the uniqueness property for the Gauss semigroups among all Gauss semigroups on simply connected nilpotent Lie groups, generalizing the corresponding result for simply connected step 2-nilpotent Lie groups by Baldi [1] but he left open the question if Gaussian measures can also be embedded into non-Gaussian c.c.s. Neuenschwander [11] showed that on the three-dimensional Heisenberg group, this is indeed not the case, i.e., every Gaussian probability measure on the three-dimensional Heisenberg group is embeddable into a unique c.c.s. The generalization of this result to all simply connected step 2-nilpotent Lie groups can be found in [13]. In fact, this result can be sharpened (by the same proof) to the uniqueness of convolution roots of Gaussian measures (recall that, in particular, on finite-dimensional vector spaces $\mathbb{R}^{d}$, all convolution roots in $M^{1}\left(\mathbb{R}^{d}\right)$ of infinitely-divisible probability measures are unique):

Proposition 1 Assume that $\mu$ is a Gaussian probability measure on the simply connected step 2-nilpotent Lie group $G$ and suppose that $\lambda$ and $\eta$ are probability measures on $G$ such that, for some $n \geq 1$, we have

$$
\lambda^{* n}=\eta^{* n}=\mu .
$$

Then $\lambda=\eta$.

Applications of c.c.s. (in particular, of Gaussian ones) on the Heisenberg group in mathematical finance (e.g., in order to model processes with stochastic volatility) are given in [15]. For the more general framework of nilpotent quantum groups and braided groups, see [4].

In the present paper, we show that this embeddability into a unique c.c.s. is valid for Gaussian probability measures on simply connected Lie groups $G$ which are nilpotent of any step $r$. The general idea of the proof is a recursive calculation of moments in order to show that all embedding c.c.s. of a Gaussian probability measure are Gaussian. Then the assertion follows from Pap's [17] afore-mentioned uniqueness result for Gaussian embedding c.c.s. As a complement, we will show how our approach can be used to give an independent proof of Pap's result itself. In this sense, our proof of the uniqueness of the embedding semigroup of a Gaussian measure can be formulated in a self-contained way.

By a similar reasoning one can also prove the uniqueness of the embedding Poisson semigroup for compound Poisson processes on simply connected nilpotent Lie groups if the jump law is determinate (which means that it possesses all absolute 
moments and that there is no other bounded nonnegative measure with the same moments). A nonnegative bounded measure on a finite-dimensional vector space $\mathbb{R}^{d}$ is of course determinate if its characteristic function (Fourier transform) is an entire function on $\mathbb{C}^{d}$. A well-known sufficient condition for determinacy is Carleman's criterion: Let $H_{j}$ be the $j$ th absolute moment of a bounded nonnegative measure on $\mathbb{R}^{d}$. Then the measure is determinate if

$$
\sum_{j=1}^{\infty} H_{2 j}^{-1 /(2 j)}=\infty .
$$

For the Gaussian case of our result, see also [14].

\section{The Result and Its Proof}

The $k$ th absolute moment of a nonnegative measure $\mu$ on a simply connected nilpotent Lie group $G$ is defined as $\int_{G}\|x\|^{k} \mu(d x)$ (where $\|$.$\| denotes the euclidean$ norm). A bounded nonnegative measure $\theta$ on $G$ is called determinate if it possesses all absolute moments and if there is no other nonnegative bounded measure on $G$ with the same "mixed" moments $H_{\ell}(\theta)=\int_{G} x_{1}^{\ell_{1}} x_{2}^{\ell_{2}} \cdots x_{d}^{\ell_{d}} \theta(d x)$ (where $\left.x=\left(x_{1}, x_{2}, \ldots, x_{d}\right) \in \mathbb{R}^{d} \cong G, \ell=\left(\ell_{1}, \ell_{2}, \ldots, \ell_{d}\right) \in \mathbb{N}_{0}^{d}\right)$.

Theorem 1 Let $\mathcal{S}^{(i)}=\left\{\mu_{t}^{(i)}\right\}_{t \geq 0}(i=1,2)$ be c.c.s. on a simply connected nilpotent Lie group $G$ such that $\mu_{1}^{(1)}=\mu_{1}^{(2)}$. Suppose that one of the following two conditions is fulfilled:

(i) The c.c.s. $\mathcal{S}^{(1)}$ is Gaussian

(ii) both c.c.s. $\mathcal{S}^{(1)}$ and $\mathcal{S}^{(2)}$ are Poissonian semigroups such that the exponent measure (or, equivalently, the law of the nonzero jumps in the nontrivial case) of $\mathcal{S}^{(1)}$ is determinate.

Then $\mu_{t}^{(1)}=\mu_{t}^{(2)}$ for all $t \geq 0$.

Let $r$ be the step of nilpotency of $G$. One of the main ingredients of the proof is the following property of recursive calculation of moments of convolution roots on a simply connected nilpotent Lie group (see [12]):

Consider an adapted vector space decomposition of $G=\mathcal{G}$, i.e.,

$$
G \cong \mathcal{G}=\mathbb{R}^{d}=\bigoplus_{i=1}^{r} V_{i}
$$

such that

$$
\bigoplus_{i=k}^{r} V_{i}=\mathcal{G}_{k-1},
$$

where $\left\{\mathcal{G}_{k}\right\}_{0 \leq k \leq r}$ is the descending central series:

$$
\mathcal{G}_{0}:=\mathcal{G}, \quad \mathcal{G}_{k+1}:=\left[\mathcal{G}, \mathcal{G}_{k}\right]
$$


(and thus $\mathcal{G}_{r}=\{0\}$ ). We take a Jordan-Hölder basis for $\mathcal{G}=\mathbb{R}^{d}$, i.e., a basis $E=$ $\left\{e_{1}, e_{2}, \ldots, e_{d}\right\}=\bigcup_{i=1}^{r} E_{i}$, where $E_{i}=\left\{e_{i, 1}, e_{i, 2}, \ldots, e_{i, d(i)}\right\}$ is a basis of $V_{i}(d(i)$ thus being the dimension of $V_{i}$ ).

Consider on $\mathbb{N}_{0}^{d}$ the lexicographic ordering from behind defined as follows: Put $\left(a_{1}, a_{2}, \ldots, a_{d}\right)<\left(b_{1}, b_{2}, \ldots, b_{d}\right)$ if $\left(a_{d}, a_{d-1}, \ldots, a_{d-j+1}\right)=\left(b_{d}, b_{d-1}, \ldots, b_{d-j+1}\right)$ and $a_{d-j}<b_{d-j}$ for some $j \in\{0,1, \ldots, d-1\}$. Consider the component decomposition $G \ni x=: \sum_{j=1}^{d} x_{j} e_{j}$.

Lemma 1 Assume that $\mu, v$ are probability measures on a simply connected nilpotent Lie group $G \cong \mathbb{R}^{d}$ satisfying $\mu=v^{* 2}$ and such that $\mu$ possesses all absolute moments. Then $v$ possesses all absolute moments, too, and the $M_{\ell}(v)\left(\ell \in \mathbb{N}_{0}^{d}\right)$ can be calculated out of the $M_{\ell}(\mu)$ recursively with respect to $\ell$.

Proof The measure $\mu$ possesses all absolute moments, and it can easily be shown (by the group property of $G$ ) that the tails of $v$ (i.e., $v(\{x \in G:\|x\| \geq \rho\})$ ) cannot have a qualitatively heavier decrease (in the sense of absolute integrability of power functions) than those of $\mu$ (as $\rho \rightarrow \infty)$. This can, e.g., be seen by integration by parts. Hence all power functions of components are absolutely $\nu$-integrable, i.e., the measure $v$ also possesses all absolute moments. Assume that $X, Y$ are i.i.d. $G$-valued random variables with law $\mathcal{L}(X)=v$. Write

$$
M_{\ell}(\mu)=E\left(\prod_{j=1}^{d}(X \cdot Y)_{j}^{\ell_{j}}\right)=E\left(\prod_{j=1}^{d}\left(X+Y+\frac{1}{2}[X, Y]+\cdots\right)_{j}^{\ell_{j}}\right) .
$$

By the adaptedness, we get, by multiplying out, the relation

$$
\left(X+Y+\frac{1}{2}[X, Y]+\cdots\right)_{j}^{\ell_{j}}=X_{j}^{\ell_{j}}+Y_{j}^{\ell_{j}}+P_{j},
$$

$P_{j}$ being a polynomial in $X_{1}, Y_{1}, X_{2}, Y_{2}, \ldots, X_{j}, Y_{j}$, where in every monomial the exponents of $X_{j}$ and $Y_{j}$ are strictly smaller than $\ell_{j}$. Now, by multiplying out the product $\prod_{j=1}^{d}(\cdots)_{j}^{\ell_{j}}$ we get

$$
\prod_{j=1}^{d}(\cdots)_{j}^{\ell_{j}}=\prod_{j=1}^{d} X_{j}^{\ell_{j}}+\prod_{j=1}^{d} Y_{j}^{\ell_{j}}+P
$$

where $P$ is a polynomial in $X_{1}, Y_{1}, X_{2}, Y_{2}, \ldots, X_{d}, Y_{d}$ with the property that, for every monomial $\prod_{j=1}^{d}\left(X_{j}^{r_{j}} Y_{j}^{s_{j}}\right)$, we have $\left(r_{1}, r_{2}, \ldots, r_{d}\right),\left(s_{1}, s_{2}, \ldots, s_{d}\right)<\ell$. Now the assertion follows from the independence of $X$ and $Y$ and from the fact that $E\left(\prod_{j=1}^{d} X_{j}^{\ell_{j}}\right)=E\left(\prod_{j=1}^{d} Y_{j}^{\ell_{j}}\right)$.

We will use the "Poisson Approximation Theorem" (or "Accompanying Laws Theorem") in the following form (see, e.g., [11, Chap. 1, Proposition 1.3]): 
Lemma 2 Let $\left\{\mu_{t}\right\}_{t \geq 0}$ be a c.c.s. on a simply connected nilpotent Lie group G. Then we have

$$
\exp \left((s / t)\left(\mu_{t}-\varepsilon_{0}\right)\right) \stackrel{w}{\rightarrow} \mu_{s} \quad(t \rightarrow 0)(s \geq 0)
$$

Together with [11, Chap. 1, Proposition 1.2], the identification of generating distributions on simply connected nilpotent Lie groups with those on the underlying vector space and the well-known convergence conditions for infinitely divisible laws on a finite-dimensional vector space (which are a generalization of those for the real line, see, e.g., [5, Theorem 19.2], and any standard literature on the subject), Lemma 2 implies Lemma 3:

Lemma 3 Assume that $\left\{\mu_{t}\right\}_{t \geq 0}$ is a c.c.s. on a simply connected nilpotent Lie group $G \cong \mathbb{R}^{d}$ with generating distribution $[\xi, M, \eta]$. Write the matrix $M=:\left(m_{i, j}\right)_{1 \leq i, j \leq d}$. Then

$$
(1 / t) \mu_{t}(B) \rightarrow \eta(B) \quad(t \rightarrow 0)
$$

(for every Borel subset $B$ of $G$ bounded away from 0 and carrying $\eta$-measure zero on its boundary). Furthermore,

$$
\limsup _{n \rightarrow \infty} 2^{n} \int_{\{x \in G:\|x\| \leq \varepsilon\}} x_{i} x_{j} \mu_{2^{-n}}(d x) \rightarrow m_{i, j} \quad(\varepsilon \rightarrow 0) .
$$

In what follows, the symbol $C$ is used for a generic finite positive constant (of possibly changing value) depending only on the fixed step $r$ of nilpotency.

Proof of Theorem 1 1. We first treat the Gaussian case. Let $\mathcal{S}^{(i)}=\left\{\mu_{t}^{(i)}\right\}_{t \geq 0}(i=1,2)$ be c.c.s. on $G$ and assume that $\mathcal{S}^{(1)}$ is Gaussian and $\mu_{1}^{(1)}=\mu_{1}^{(2)}$. By the abovementioned uniqueness result for the embedding Gaussian c.c.s. of a Gaussian measure due to Pap [17], it suffices to show that $\mathcal{S}^{(2)}$ also is Gaussian. Define the bounded nonnegative measures $\eta_{t}^{(i)}:=(1 / t) \mu_{t}^{(i)}$ and (for Borel subsets $B$ of $G$ ) the nonnegative measures

$$
\iota_{t}^{(i)}(B):=\int_{B}\|x\|^{4} \eta_{t}^{(i)}(d x)
$$

(a priori it is not excluded that $\iota_{t}^{(i)}(B)=\infty$ for certain Borel sets $B$ ). Let $Z=$ $\int_{0}^{1} \int_{0}^{s_{m}} \cdots \int_{0}^{s_{2}} d B_{1}\left(s_{1}\right) d B_{2}\left(s_{2}\right) \cdots d B_{m}\left(s_{m}\right)$ be an iterated stochastic integral of $m$ $(m \leq r)$ one-dimensional standard Brownian motions which are (for every pair $(i, j)$ of coordinates) either independent or identical. If we assume that all occurring onedimensional Brownian motions are independent, we can directly invoke a result in [18] giving the asymptotic behavior of the density $f(x)$ of $Z$ in the case of $m$ independent $B_{i}$ 's:

$$
f(x)=O\left(\exp \left(-C|x|^{2 / m}\right)\right) \quad(x \rightarrow \infty) .
$$


This implies that, for $x$ large enough and a constant $C^{\prime}>0$ small enough, we have the tail behavior

$$
\begin{aligned}
P(|Z| \geq x) & \leq \int_{x}^{\infty} y^{(2 / m)-1} \exp \left(\left(C^{\prime}\right) y^{2 / m}\right) f(y) d y \\
& \leq \exp \left(-C x^{2 / m}\right) \leq \exp \left(-C x^{2 / r}\right) .
\end{aligned}
$$

In the case that some of these one-dimensional Brownian motions are identical, additional terms $d B_{i} \cdot d B_{i}=d t$ arise in the evaluation of the iterated stochastic integral $Z$. One can easily see that they do not disturb the qualitative tail behavior.

Since multi-dimensional centered Brownian motions on the vector space $\left(\mathbb{R}^{d},+\right)$ can always be written as linear transformations of a multi-dimensional standard Brownian motion (i.e., one with independent one-dimensional standard Brownian motions as components), it follows that any one-dimensional component $T$ of a Gaussian random variable on $G$ has the form of a linear combination of expressions of the form $Z$ (with $m \leq r$ ). By the scaling property of the one-dimensional standard Brownian motion $\{B(t)\}_{t>0}$ (which means that $\{B(u t)\}_{t>0}$ has the same law as $\left\{u^{1 / 2} B(t)\right\}_{t \geq 0}$ for $\left.u>0\right)$, one sees at once that the above-mentioned " $(2 / r)$ exponential tail decrease" (1) of $Z$ (applied to $\mu_{1}^{(1)}=\eta_{1}^{(1)}$ ) holds uniformly in time $t \in[0,1]$ for the measures $\eta_{t}^{(1)}$, since if $Z_{t}$ denotes a similar iterated stochastic integral as $Z$ but (in contrast to $Z$ ) evaluated between times 0 and $t$ (instead of 1 ) of $m$ one-dimensional standard Brownian motions, then $Z_{t}$ has the same law as $t^{m / 2} Z_{1}$ (also in the case with several identical one-dimensional Brownian motions). Using Lemma 3 and the just proved uniform $(2 / r)$-exponential tail decrease (which implie the uniform integrability of the fourth absolute moments of the measures $\eta_{t}^{(1)}$ for $t \leq 1)$, we obtain from the Gaussianity of $\mathcal{S}^{(1)}$ that $\iota_{t}^{(1)}(G) \rightarrow 0(t \rightarrow 0)$. By Lemma 1 the measures $\mu_{2^{-n}}^{(2)}$ also possess all absolute moments and, moreover, for every fixed $n$, $\ell$, we have that $M_{\ell}\left(\mu_{2^{-n}}^{(1)}\right)=M_{\ell}\left(\mu_{2^{-n}}^{(2)}\right)$. Thus also $\iota_{2^{-n}}^{(2)}(G) \rightarrow 0(n \rightarrow \infty)$. By Lemma 3 it follows that indeed $\mathcal{S}^{(2)}$ has to be Gaussian, too, which finishes the proof of Theorem 1 for the Gaussian case.

2. Let us now turn to the Poissonian situation. We keep the notation of part 1 of the proof. In particular, we define the bounded nonnegative measures $\eta_{t}^{(i)}:=(1 / t) \mu_{t}^{(i)}$ and (for Borel subsets $B$ of $G$ ) the nonnegative measures

$$
\kappa_{t}^{(i)}(B):=\int_{B}\|x\|^{2} \eta_{t}^{(i)}(d x),
$$

(also here, a priori it is not excluded that $\kappa_{t}^{(i)}(B)=\infty$ for certain Borel sets $B$ ). Suppose (in contrast to part 1) that $\left\{\mathcal{S}^{(i)}\right\}$ are Poisson semigroups on $G$ and that the exponent measure $\eta^{(1)}$ of $\mathcal{S}^{(1)}$ is determinate. We first must show that the measures $\eta_{2^{-n}}^{(1)}=2^{n} \mu_{2^{-n}}^{(1)}$ possess all absolute moments and that for every fixed $j \in \mathbb{N}$, the absolute $j$-integrability of the tails of $\eta_{2^{-n}}^{(1)}$ is uniform in $n$. W.l.o.g. we may assume $\eta^{(i)} \neq 0(i=1,2)$. Define $\bar{\eta}^{(i)}:=\eta^{(i)} /\left\|\eta^{(i)}\right\|(\|$.$\| denoting the total variation norm).$ At the beginning, let us have a look at the convolution powers $\left(\bar{\eta}^{(1)}\right)^{* k}$. Take i.i.d. $G$-valued random variables $X_{n}$, each one obeying the law $\bar{\eta}^{(1)}$, and consider their 
products $\prod_{i=1}^{k} X_{i}$. Observe that in the "iterated" Campbell-Hausdorff formula for the product of $k$ factors, the number of occurring "standard Lie bracket monomials" (i.e., compositions of mappings $\left.a d\left(X_{i}\right)\right)$ can be estimated from above by $(k+1)^{r}$. On the other hand, if we want to estimate a $j$ th absolute moment of such a $k$-fold group product (i.e., $E\|(\cdots)\|^{j}$, where in the parentheses, there is a group product of $k$ random variables), then we can first develop the latter product $(\cdots)$ with respect to the (iterated) Campbell-Hausdorff formula for $k$ factors as before, and then the $j$ th power of the norm (i.e., the term $\|(\cdots)\|^{j}$ ) by the multinomial theorem. So, for the $j$ th absolute moments, this yields (counted with the corresponding multiplicities given by the multinomial coefficients) at most $(k+1)^{r j}$ summands (norms of standard Lie bracket monomials as described before). Now by the Dominated Convergence Theorem and by using the Cauchy-Schwarz inequality for the Lie bracket we obtain (for fixed $j$ and fixed $n$ ) a finite upper estimate for the $j$ th absolute moments of $\eta_{2^{-n}}^{(1)}$ and (for fixed $j$ ) also the uniform $j$-integrability of the tails of $\eta_{2^{-n}}^{(1)}$ for $n \in \mathbb{N}_{0}$. Now, by analogy with part 1 , it follows that all mixed moments of $\kappa_{2-n}^{(1)}$ tend to those of the nonnegative measure $\kappa^{(1)}$, where $\kappa^{(i)}(B):=\int_{B}\|x\|^{2} \eta^{(i)}(d x)$ ( $B$ a Borel subset of $G$ ). From Lemma 1 it follows that, for every $j$, the measures $\kappa_{2^{-n}}^{(2)}$ are uniformly $\mathcal{L}^{j}$-bounded. So it follows from the determinacy of $\kappa^{(1)}$, together with Lemma 3 and a classical "diagonal subsequence" argument, that for any subsequence $\left\{n^{\prime}\right\} \subset\{n\}$, there is a sub-subsequence $\left\{n^{\prime \prime}\right\} \subset\left\{n^{\prime}\right\}$ such that $\kappa_{2^{-n^{\prime \prime}}}^{(2)} \stackrel{w}{\rightarrow} \kappa^{(1)}$ (sic!) $\left(n^{\prime \prime} \rightarrow \infty\right)$. Hence we have that $\kappa_{2^{-n}}^{(2)} \stackrel{w}{\rightarrow} \kappa^{(1)}$ as $n \rightarrow \infty$. So $\kappa^{(1)}=\kappa^{(2)}$, which entails $\eta^{(1)}=\eta^{(2)}$.

\section{Another Proof of Pap's Uniqueness Result for Gaussian Embedding Semigroups on Simply Connected Nilpotent Lie Groups}

Remark 1 In this little appendix, we show how the above approach of the proof of sufficiency of condition (i) in Theorem 1 can be directly used to give an independent direct proof of Pap's [17] result on the uniqueness of the Gaussian embedding semigroup of a Gaussian probability measure on a simply connected nilpotent Lie group. In this sense, the proof of the first part of Theorem 1 can be kept quite self-contained. We will keep the notation of the proof of Theorem 1. For $i=1,2$, let $\mathcal{S}^{(i)}=\left\{\mu_{t}^{(i)}\right\}_{t \geq 0}$ be Gaussian c.c.s. on a $d$-dimensional simply connected nilpotent Lie group $G$ such that $\mu_{1}^{(1)}=\mu_{1}^{(2)}$. Define the signed measures

$$
\kappa_{k, \ell ; t}^{(i)}(B):=\int_{B} x_{k} x_{\ell} \eta_{t}^{(i)}(d x)
$$

for Borel subsets $B$ of $G$ (observe that the above integral indeed converges absolutely due to the tail decrease of Gaussian measures on $G$ verified in the proof of the sufficiency of condition (i) of Theorem 1). By the above-mentioned convergence conditions for c.c.s. on Lie groups, it follows (similarly as for the convergence of $\iota_{t}^{(i)}$ in the verification of the sufficiency of condition (i) in Theorem 1) that

$$
\kappa_{k, \ell ; 2^{-n}}^{(i)}(B) \rightarrow m_{k, \ell}^{(i)} \quad(n \rightarrow \infty)
$$


for every Borel subset $B$ of $G$ containing 0 in its interior, where $M^{(i)}=:\left(m_{k, \ell}^{(i)}\right)_{1 \leq k, \ell \leq d}$ denotes the positive semidefinite $(d \times d)$-matrix yielding the centered Gaussian part in the Lévy-Hinčin formula of the c.c.s. $\mathcal{S}^{(i)}$. So, by an analogous argumentation as above about the recursive calculability of the mixed moments, it follows that $M^{(1)}=M^{(2)}$. If $\xi^{(i)}$ represents the primitive (shift) term in the Lévy-Hinčin formula of $\mathcal{S}^{(i)}$, then we get that $\xi^{(1)}=\xi^{(2)}$ by induction on the step $r$ of nilpotency; for the induction step $r \rightarrow r+1$, look at the projection onto the simply connected step $r$-nilpotent quotient Lie group (or rather quotient Lie algebra) $\mathcal{G} / \mathcal{G}_{r}$ and use the fact that on a finite-dimensional vector space $V$, for a nondegenerate probability measure $\mu$ on $V$, we can only have $\mu * \varepsilon_{x}=\mu * \varepsilon_{y}$ if $x=y$.

\section{References}

1. Baldi, P.: Unicité du plongement d'une mesure de probabilité dans un semi-groupe de convolution Gaussien. Cas non-Abélien. Math. Z. 188, 411-417 (1985)

2. Böge, W.: Ueber die Charakterisierung unendlich teilbarer Wahrscheinlichkeitsverteilungen. J. Reine Angew. Math. 201, 150-156 (1959)

3. Burrell, Q.L., McCrudden, M.: Infinitely divisible distributions on connected nilpotent Lie groups. J. London Math. Soc. (2) 7, 584-588 (1974)

4. Franz, U., Neuenschwander, D., Schott, R.: Gauss laws in the sense of Bernstein and uniqueness of embedding into convolution semigroups on quantum groups and braided groups. Prob. Theory Rel. Fields 109, 101-127 (1997)

5. Gnedenko, B.V., Kolmogorov, A.N.: Limit Distributions for Sums of Independent Random Variables. Addison-Wesley, Cambridge (1954)

6. Graczyk, P.: Cramér theorem on symmetric spaces of noncompact type. J. Theor. Probab. 7(3), 609613 (1994)

7. Hazod, W.: Ueber Wurzeln und Logarithmen beschränkter Masse. Z. Wahrscheinlichkeitstheorie verw. Geb. 20, 259-270 (1971)

8. Hazod, W., Scheffler, H.P.: The domains of partial attraction of probabilities on groups and on vector spaces. J. Theor. Probab. 6(1), 175-186 (1993)

9. Hazod, W., Siebert, E.: Stable Probability Measures on Euclidean Spaces and Locally Compact Groups. Structural Properties and Limit Theorems. Kluwer Academic, Dordrecht (2001)

10. Heyer, H.: Probability Measures on Locally Compact Groups. Springer, Berlin (1977)

11. Neuenschwander, D.: Probabilities on the Heisenberg Group: Limit Theorems and Brownian Motion. Lecture Notes in Mathematics, vol. 1630. Springer, Berlin (1996)

12. Neuenschwander, D.: On the uniqueness problem for continuous convolution semigroups of probability measures on simply connected nilpotent Lie groups. Publ. Math. Debrecen 53(3-4), 415-422 (1998)

13. Neuenschwander, D.: s-stable semigroups on simply connected step 2-nilpotent Lie groups. In: Budzban, G., et al. (eds.) Probability on Algebraic Structures, AMS Special Session, Gainesville, FL, USA, March 12-13, 1999. AMS, Providence (2000). Contemp. Math. 261, 59-70

14. Neuenschwander, D.: Uniqueness of embedding of Gaussian probability measures into a continuous convolution semigroup on simply connected nilpotent Lie groups. C. R. Acad. Sci. Paris (2008, to appear)

15. Neuenschwander, D.: Retrieval of Black-Scholes and generalized Erlang models by perturbed observations at a fixed time. Insur.: Math. Econ. 42/1, 453-458 (2008)

16. Nobel, S.: Limit theorems f-284 (1991)

17. Pap, G.: Uniqueness of embedding into a Gaussian semigroup on a nilpotent Lie group. Arch. Math. (Basel) 62, 282-288 (1994)

18. Schott, R.: Une loi du logarithme itéré pour certaines intégrales stochastiques. C. R. Acad. Sci. Paris Sér. I 292, 295-298 (1981)

19. Serre, J.-P.: Lie Algebras and Lie Groups. Benjamin, New York (1965)

20. Siebert, E.: Ueber die Erzeugung von Faltungshalbgruppen auf beliebigen lokalkompakten Gruppen. Math. Z. 131, 313-333 (1973)

21. Siebert, E.: Fourier analysis and limit theorems for convolution semigroups on a locally compact group. Adv. Math. 39, 111-154 (1981) 